ESJ Natural/Life/Medical Sciences

\title{
Epidémiologie De La Mortalité En Réanimation Polyvalente De l'Hôpital National De Zinder Au Niger
}

\author{
Magagi Amadou
}

Service d'anesthésie réanimation de l'hôpital national de Zinder, Niger Egbohou Pilaimwé

Service d'anesthésie réanimation du CHU Sylvanus Olympio de Lomé, Togo

\section{Maikassoua Mamane}

Boukari Mahamane Bawa

Service d'anesthésie réanimation de l'hôpital de référence de Maradi, Niger Service d'anesthésie réanimation hôpital national de Niamey, Niger

Doi:10.19044/esj.2021.v17n21p142

Submitted: 08 January 2021

Accepted: 27 May 2021

Published: 30 June 2021
Copyright 2021 Author(s)

Under Creative Commons BY-NC-ND 4.0 OPEN ACCESS

Cite As:

Magagi A., Egbohou P., Maikassoua M. \& Boukari M.B. (2021). Epidémiologie De La Mortalité En Réanimation Polyvalente De l'Hôpital National De Zinder Au Niger. European Scientific Journal, ESJ, 17(21), 142.

https://doi.org/10.19044/esj.2021.v17n21p142

\section{Résumé}

Objectif : décrire les aspects épidémiologiques des patients décédés Réanimation Polyvalente (RP) de l'hôpital Nationale de Zinder (HNZ) au Niger.

Patients et méthodes: Il s'agissait d'une étude descriptive, transversale à collecte rétrospective des données sur une période de12 mois allant du 1er janvier au 31 décembre 2019. L'étude s’est déroulée dans le service de réanimation polyvalente de l'hôpital national de Zinder au Niger. Etaient inclus dans cette étude tous les patients admis durant la période d'étude dans ce service en accord avec leurs familles et celui du comité éthique.

Résultats : Durant la période d'étude 1000 patients ont été enregistrés dont 148 décès soit un taux de mortalité de 14,8 \%. L’âge moyen était de 36,45 ans avec une prédominance de sexe masculin dans 56,1\% (83 hommes). Les taux de décès étaient plus élevés durant les mois de mars, juin et aout avec respectivement $19,8 \% 17 \%$ et $22,2 \%$. Les étiologies des décès étaient médicales dans 54\% des cas dont l'AVC (18,91 \%) et le diabète (13,51 \%). 
Les causes chirurgicales étaient responsables de $46 \%$ des décès, dont $23 \%$ par péritonite aiguë généralisée. Près de la moitié des décès $(49,2 \%)$ survenaient dans les 24 premières heures.

Conclusion : le taux de mortalité en RP de HNZ était élevé. Les pathologies médicales étaient responsables de plus de décès que celles chirurgicales. Des efforts d'amélioration du plateau technique de la RP, des services d'aide au diagnostic et à la prise en charge de l'hôpital national de Zinder, et une sensibilisation à une consultation précoce réduiraient certainement ce taux.

Mots clés: Épidémiologie, Mortalité, Réanimation, Niger

\section{Epidemiology Of Mortality In Multipurpose Intensive Care Unit At The Zinder National Hospital In Niger}

\section{Magagi Amadou}

Service d'anesthésie réanimation de l'hôpital national de Zinder, Niger Egbohou Pilaimwé

Service d'anesthésie réanimation du CHU Sylvanus Olympio de Lomé, Togo Maikassoua Mamane Boukari Mahamane Bawa

Service d'anesthésie réanimation de l'hôpital de référence de Maradi, Niger Service d'anesthésie réanimation hôpital national de Niamey, Niger

\section{Abstract}

Objective: to describe the epidemiological characteristics of patients dying in the multipurpose intensive care unit (M ICU) at Zinder National Hospital (ZNH) in Niger.

Patients and method: it was a cross-sectional descriptive study over 12-months, from January 1 to December 31, 2019. The study was conducted in the MICU at the ZNH. All patients admitted to this MICU during the study period were included. Deaths were recorded and analyzed.

Results: A total of 148 deaths out of 1000 admissions was recorded, mortality rate: $14.8 \%$. The average age was 36.45 years old. The male predominated: 83 (56.1\%) deaths. Death rates were higher during the months of August (22.2\%), March (19.8\%), June (17.4\%) and September (17\%). Medical causes of deaths accounted for $54 \%$, with stroke (18.91\%), followed by diabetes metabolic complications (13.51\%). Surgical causes accounted for $46 \%$ of deaths, with $23 \%$ of acute generalized peritonitis. Nearly half of the deaths (49.2\%) occurred within the first 24 hours. 
Conclusion: The mortality rate in MICU at ZNH was high. Medical conditions were responsible for more deaths than surgical ones. Efforts to improve the MICU facilities, diagnostic and support services at ZNH, and early consultation awareness would surely reduce this rate.

Keywords: Epidemiology, Mortality, Resuscitation, Niger

\section{Introduction}

La réanimation est une discipline médicale ayant pour mission de prendre en charge des patients présentant ou susceptibles de présenter des défaillances viscérales aiguës dont le pronostic à court terme est mis en jeu. Les taux de décès malgré des moyens thérapeutiques lourds mis en jeu pour suppléer ces fonctions défaillantes sont souvent élevés. Des études dans des pays africains rapportent des taux de mortalité élevés variant de 7,8\% au Cameroun à 51,6 \% au Burkina Faso, avec des étiologies aussi bien médicales que chirurgicales [1-5]. Notre étude avait pour objectif de décrire les aspects épidémiologiques des patients décédant en Réanimation Polyvalente (RP) de l’hôpital Nationale de Zinder (HNZ) au Niger.

\section{Patients et méthodes}

Il s'agissait d'une étude descriptive à collecte rétrospective des données sur une période de12 mois allant du $1^{\mathrm{er}}$ janvier au 31 décembre 2019. L'étude s'était déroulée dans le service de réanimation polyvalente (RP) de l'hôpital national de Zinder (HNZ) au Niger. C'est une réanimation polyvalente mixte médicale et chirurgicale de 15 lits. Elle reçoit les patients de tout sexe et tout âge. Ce service est le principal centre de référence en matière de réanimation pour les régions de Zinder, Maradi, Diffa, Agadez et d'une partie du Nord du Nigeria. Les soins y sont assurés par un médecin anesthésiste réanimateur et 14 infirmiers diplômés d'Etat. Comme équipement le service disposait de deux (2) respirateurs et un moniteur de surveillance pour chaque lit. Les bilans biologiques de routine (détermination de groupe sanguin $\mathrm{ABO} / \mathrm{Rh}$, numération sanguine, créatinémie, glycémie, ionogramme sanguin) étaient disponibles, de même que des radiographies standards et un scanner. Il n'existait pas à Zinder un service de prise en charge pré hospitalier des traumatisés. Etaient inclus dans cette étude les patients admis directement au service de réanimation, ceux provenant d'autres services de l'HNZ et les référés des structures sanitaires des régions suscitées tous décédés au service de réanimation. Une fiche a été utilisée pour le recueil des données auprès des patients conscients et des accompagnants lorsque les patients étaient inconscients. Les variables étudiées étaient démographiques (l'âge, le sexe, le motif d'admission, la provenance), l'état clinique à l'admission, la durée d’hospitalisation, les causes du décès. Les données ont 
été analysées avec le logiciel Statistical Package for Social Science (SPSS) version 21. Les variables qualitatives ont été exprimées sous forme de fréquences et pourcentages et celles quantitatives sous forme de moyennes.

\section{Résultats}

Les patients admis en réanimation étaient souvent dans des états cliniques très préoccupant dont parfois le pronostic était réservé. Cet état clinique fragile à l'admission était souvent source de décès. Durant cette étude mille (1000) patients ont été enregistrés et 148 d'entre eux n’ont pas survécu soit un taux de mortalité de 14,8\%. L’âge moyen des patients décédés était de 36,45 ans avec des extrêmes de 02 jours à 85 ans. Le sexe masculin prédominait avec $83(56,1 \%)$, le sexe ratio était de 1,27 . Les mois de septembre, octobre et novembre ont enregistré plus d'admission avec respectivement 100, 107 et 98 patients. Les taux de décès étaient plus observés durant les mois de mars, juin, aout et septembre avec respectivement 19,8\% $17,4 \% 22,2 \%$ et $17 \%$ des cas. Les étiologies des décès étaient médicales (AVC, diabète) dans $54 \%$ soit 80 patients et chirurgicales (péritonite, traumatisme) dans $46 \%$ soit 68 admis.

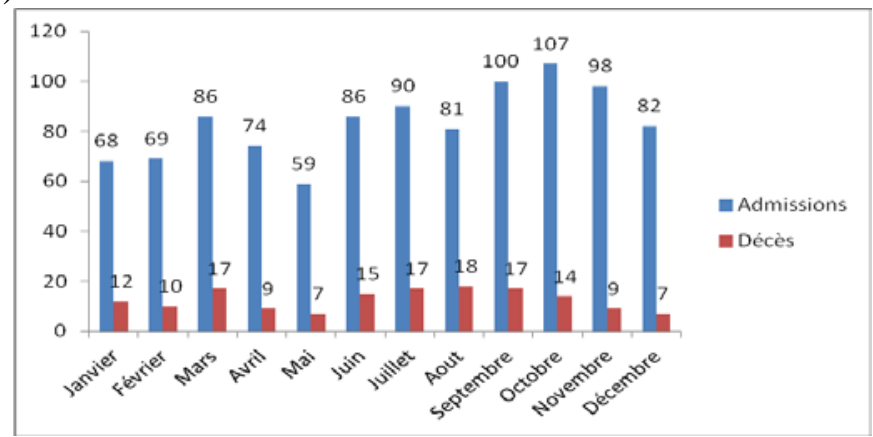

Figure 1. Répartition mensuelle des admissions et des décès

La figure $\mathrm{n}^{\circ} 1$ illustre les admissions et les décès au cours des différents mois de l'année d'étude. Aux mois de mars , juin , juillet, aout et septembre nous avions observés plus de décès avec respectivement $17,15,17,18$ et 18 cas de décès.

Tableau 1. Causes médicales des décès

\begin{tabular}{|l|l|l|}
\hline Pathologies & Nombre de cas de décès & Pourcentage \\
\hline AVC $^{1}$ & 28 & $18,91 \%$ \\
\hline Diabète & 20 & $13,51 \%$ \\
\hline Insuffisance rénale & 06 & $04,05 \%$ \\
\hline Anémie sévère & 03 & $02,02 \%$ \\
\hline Paludisme grave & 05 & $03,37 \%$ \\
\hline Etat de mal convulsif & 03 & $02,02 \%$ \\
\hline Coma & 10 & $06,75 \%$ \\
\hline Détresse respiratoire & 05 & $03,37 \%$ \\
\hline
\end{tabular}

1: AVC = accident vasculaire cerebral 
La répartion des patients selon les causes médicales du décès ressortait que l'accident vasculaire cérébral ou AVC occupait la première place avec 28 décès soit $18,91 \%$, suivi des complications du diabète avec 20 décès soit $13,51 \%$.

Tableau 2. Causes chirurgicales des décès

\begin{tabular}{|l|l|l|}
\hline Pathologie & Nombre de cas & Pourcentage \\
\hline Péritonite aiguë généralisée & 35 & $23,64 \%$ \\
\hline Occlusion intestinale & 02 & $01,35 \%$ \\
\hline Malformation ano -rectale & 04 & $02,70 \%$ \\
\hline Hernie ombilicale et inguinale & 03 & $02,02 \%$ \\
\hline Invagination intestinale & 03 & $02,02 \%$ \\
\hline Appendicite aiguë & 01 & $00,67 \%$ \\
\hline Tumeur abdominale & 03 & $02,02 \%$ \\
\hline Traumatisme grave & 12 & $08,10 \%$ \\
\hline Brulure sévère & 04 & $02,70 \%$ \\
\hline Hypertrophie bénigne de la prostate & 01 & $00,67 \%$ \\
\hline
\end{tabular}

Le tableau $\mathrm{n}^{\circ} 2$ présentait le détail des étiologies chirurgicales enregistrées comme cause des décès. La péritonite aiguë généralisée était la pathologie chirurgicale venant en tête des décès avec 34 (23,6\%) décès. Elle était suivie des traumatismes graves avec 12 décès $(08,10 \%)$. Le tableau 2 détaille les étiologies chirurgicales des décès survenus en RP. Près de la moitié des décès $(49,2 \%)$ survenaient dans les 24 premières heures.

\section{Discussion}

Au cours de la période d'étude 1000 patients ont été admis en RP parmi lesquels 148 étaient décédés soit un taux de 14,8\%. Ce taux de décès était plus élevé que celui de Metogo Mbengono et al au Cameroun de 7,8 \% ou de Coulibaly et al à Bamako au Mali avec 5,2 \% [4, 6]. Il est par contre plus bas que ceux trouvés ailleurs au Togo, au Mali, au Maroc, ou au Burkina Faso avec des taux de mortalité en réanimation respectifs de 32,4\%, 41,46 \%, 25,8 $\%, 51,6 \%[1-3,5]$. Le taux élevé de décès dans cette étude trouvait son explication dans la sévérité des pathologies impliquées, l'insuffisance de moyen et surtout le retard à la consultation des patients. Le sexe masculin était prédominant avec 56,08 \%. Ce même constat a été fait dans les études suscitées de Bonkoungou et al au Burkina, Egbohou et al au Togo avec respectivement 56,7 \% et 68,8 \% [1,5]. Par contre Metogo Mbengono et al au Cameroun et Sougane et al au Mali avaient enregistrés plus des femmes avec respectivement $52,1 \%$ et $52,3 \%$ [3, 4]. Cette prédominance féminine de certaines séries s'expliquerait par le fait que les femmes seraient majoritaires dans la population générale des pays d'étude. Quant à la prédominance masculine, elle trouverait son explication à la plus grande exposition des hommes surtout aux traumatismes, les femmes étant confinées au domicile et 
donc moins exposées. Dans notre série l’âge moyen des patients décédant était de 36,45 ans avec des extrêmes de 02 jours à 85 ans. Cet âge jeune a été retrouvé dans d'autres études africaines; Egbohou et al à Lomé au Togo trouvaient une moyenne de 41,7ans et une prédominance de la tranche d'âge de 31 - 40 ans qui représentait 19,8 \%. Bonkoungou et al au Burkina, Metogo et al au Cameroun et Sougane et al au Mali retrouvaient des moyennes respectives de 42,9 ans, 42 ans et 45,9 ans $[\mathbf{2}, \mathbf{5}, \mathbf{8}, \mathbf{1 3}]$. Les décès étaient plus en rapport avec des pathologies médicales que chirurgicales avec dans cette série $54,1 \%$ de décès de causes médicales. De nombreuses études ont retrouvées la même prédominance léthale des pathologies médicales [3-5]. Si dans cette série l'accident vasculaire cérébral (AVC) suivi des complications métaboliques du diabète venaient en tête des pathologies médicales, les insuffisances respiratoires, les AVC, et les sepsis venaient en tête des pathologies médicales dans la série de Egbohou et al à Lomé [5].Au sein des pathologies chirurgicales, les péritonites $(23,4 \%)$ suivies des traumatismes $(8,1 \%)$ constituaient les premières causes de décès dans cette étude. Ailleurs c'étaient des pathologies traumatiques qui occupaient le devant de la scène. A Lomé Egbohou et al avait enregistré 45,15 \% cas de traumatismes dont 34,18 \% de traumatismes crâniens graves [5]. Des études en Afrique au Sud du Sahara ont rapporté, une mortalité périopératoire par des urgences abdominales élevée et identifiaient comme raisons : le retard à la consultation, la prise en charge en phase de sepsis sévère, ou de choc septique, avec installations des défaillances multi viscérales et prise en charge inadéquate [7-10]. Les traumatismes comme rapporté ailleurs, souffrent de l'absence d'une prise en charge pré hospitalière et d'une organisation inefficiente des services d'urgences à Zinder, les blessés étant transporté dans des conditions non sécuritaire par la population et sans aucune prise en charge précoce des détresses vitales, conduisant à l'aggravation de lésions traumatiques, et à l'installation d'agressions secondaires délétères notamment l'hypotension et l'hypoxie grevant le pronostic des traumatisés crâniens [5, 11].Nous avions enregistré plus de décès entre le premier et le troisième jour d'hospitalisation avec près de la moitié des décès (49,3\%) dans les premières 24 heures. Des décès en phase précoce ont été également enregistrés au Togo, 55,7 \% de décès survenant dans les 3 premiers jours, au Burkina Faso, $61 \%$ de décès dans les 72 heures, et au Mali, $73 \%$ de décès dans les 5 premiers jours [1,3, 5]. La sévérité des défaillances, le retard à l'admission et l'insuffisance du plateau technique (matériel, personnel qualifié) sont des raisons pouvant expliquer ces décès précoces.

\section{Conclusion}

De cette étude, il ressortait que les pathologies médicales étaient les plus létales avec l'AVC et les complications du diabète en tête. Les 
pathologies chirurgicales étaient essentiellement représentées par les péritonites et les traumatismes graves. Le taux élevé de décès en rapport certainement avec un plateau technique insuffisant, un retard à la consultation suggère des efforts d'équipement de la RP mais aussi de services de support connexes tels les laboratoires, l'imagerie médicale indispensable à la prise en charge, pour espérer une réduction des taux de mortalité.

\section{References:}

1. Bonkoungou P, Traore' I, Bako YP, Sanou J, Ouédraogo N. La mortalité en réanimation polyvalente du centre hospitalier universitaire Yalgado Ouédraogo de Ouagadougou au Burkina-Faso (Résumé). Ann Fr d'Anest Réanim 2014 ; 33 : A310.

2. Afra K. Facteurs de mortalite' en re'animation. [The `se en médecine, Marrakech (Maroc)], 2010. p. 23.

3. Sougane M. Mortalité et morbidité au service de réanimation du CHU de l'Hôpital Gabriel Toure de Bamako. [The`se en me'decine, Bamako (Mali)], 2006. p. 39.

4. Metogo Mbengono JA, Bengono R, Mendimi Nkodo J, et al. Étiologies des décès dans les services d'urgences et de réanimation dans deux hôpitaux de la ville de Yaoundé. Health Sci Dis 2015 ; 16 : 1-4.

5. Egbohou P, Sama HD, Mouzou T, Assenouwe S, Akala-Yoba G, Tchetike $\mathrm{F}$, Tomta $\mathrm{K}$. Etiologies des décès dans le service de réanimation polyvalente du CHU Sylvanus Olympio de Lomé, Togo. Med Sante Trop 2018 ; 28 : 281-284. doi : 10.1684/mst.2018.0823.

6. Coulibaly. Y, Goita .D, Dicko .H, Diallo.D, Keita, Doumbia.D Diallo.Morbidité et mortalité maternelle en réanimation en milieu tropical. Rev Afr Anesth Reanim et Med Urgence, 2011. Tome 16. 7. Assouto P, Tchaou B, Kangni N, et al. Evolution postopératoire précoce en chirurgie digestive en milieu tropicale. Med Trop 2009; 69 : 477-9.

7. Ayite A, Dosseh DE, Katakoa G, Tekou HA, James K. Traitement chirurgical des perforations non traumatiques uniques de l'intestin grêle : excision-suture ou résection-anastomose. Ann chir 2006 ; 131 : 91-5.

8. Kassegne I, Dosseh DE, Sewa EV, et al. E'volution postope'ratoire des laparotomies dans le service de chirurgie ge'ne'rale du centre hospitalier préfectoral D’Ane'ho (Togo). J Afr Chir Digest 2012 ; 12 : 1313-8.

9. Harouna Y, Ali L, Seibou A, et al. Deux ans de chirurgie digestive d'urgence a` l'hôpital national de Niamey (Niger) : étude analytique et pronostique. Med Afr Noire 2001 ; 48 : 49-54. 
10. McHugh GS, Engel DC, Butcher I, et al. Prognostic value of secondaryinsults in traumatic brain injury: results from the IMPACT study. J Neurotrauma 2007 ; 24 : 287-93. 